\title{
Pair-correlation in Bose-Einstein Condensate and Fermi Superfluid of Atomic Gases
}

\author{
Bimalendu Deb \\ Physical Research Laboratory, Navrangpura, Ahmedabad 380 009, India
}

\begin{abstract}
We describe pair-correlation inherent in the structure of many-particle ground state of quantum gases, namely, Bose Einstein condensate and Cooper-paired Fermi superfluid of atomic gases. We make a comparative study on the pair-correlation properties of these two systems. We discuss how to probe this pair-correlation by stimulated light scattering. This intrinsic pair-correlation may serve as a resource for many-particle entanglement.
\end{abstract}

PACS numbers: 03.75.Ss,74.20.-z,32.80.Lg 
Pair-correlation in Bose-Einstein Condensate and Fermi Superfluid of Atomic Gases 2

\section{Introduction}

The realization of Bose-Einstein condensation in dilute atomic gases [1] a decade ago marked a breakthrough revitalizing many areas of physics, particularly, atomic and molecular physics. One of the most significant advantages of experimentation with cold atoms is the ability to tune atom-atom interaction over a wide range by a magnetic field Feshbach resonance. This provides an unique opportunity to explore physics of interacting many-particle systems in a new parameter regime. In this context, cold atoms obeying Fermi-Dirac statistics have currently attracted enormous research interest. Fermions are the basic constituents of matter, therefore research with trapped Fermi atoms 2, 3, 4, 5] under controllable physical conditions has important implications in materials science. In particular, it has significant relevance in the field of superconductivity.

The first achievement of quantum degeneracy in Fermi gas of ${ }^{40} \mathrm{~K}$ atoms by Colorado group [2] in 1999 marked a turning point in the research with cold atoms. Since then, cold Fermi atoms have been of prime research interest in physics today. In a series of experiments, several groups [3, 4, 6, 7, 8, 9] have demonstrated many new features of degenerate atomic Fermi gases. In a recent experiment, Ketterle's group [5] has realized quantized vortices as a signature of Fermi superfluidity in a trapped atomic gas. Two groups [10, 11] have independently reported the measurement of pairing gap in Fermi atoms. Collective oscillations [12, 13] which are indicative of the occurrence of Fermi superfluidity [14] have been previously observed. The crossover [15, 16, 17] between BCS state of atoms and BEC of molecules formed from Fermi atoms has become a key issue of tremendous research interest. Several groups have achieved BEC [18] of molecules formed from Fermi atoms. There have been several other experimental [19] and theoretical investigations [20] on various aspects of interacting Fermi atoms.

Both atomic BEC and superfluid atomic Fermi gas have some common quantum features: (a) both are macroscopic quantum objects (b) the thermal de-Broglie wavelength greatly exceeds the interparticle separation; (c) both have off-diagonal long range order (ODLRO) or coherence; (d) the ground state of both the systems has a structure whose constituents include pair-correlated states; (e) both have ground state of broken symmetry; (f) both must possess long wave-length phonon modes for restoration of symmetry that is broken by their respective ground state. Our focus here would be the common feature (d) to investigate how this pair-correlation can be probed.

In the next section, we make a comparative study between BEC and BCS ground states. Our objective is to show that a nontrivial pair-correlation naturally arises in BEC 21 and BCS matter, and possibly it is a generic feature of all macroscopic quantum objects. In section 3, we discuss briefly some relevant features of trapped Fermi gas. In subsequent sections, we describe stimulated light scattering as a means of probing Cooper-pairing. We find that using stimulated scattering of circularly polarized light, it is possible to scatter selectively either partner atom of a Cooper-pair [22]. In the low momentum transfer regime, this may be useful in exciting Anderson-Bogoliubov phonon 
Pair-correlation in Bose-Einstein Condensate and Fermi Superfluid of Atomic Gases 3 mode of broken symmetry.

\section{A comparison between BEC and BCS states}

Bose-Einstein condensate (BEC) of a weakly interacting Bose gas and Bardeen-CooperSchrieffer (BCS) state of an interacting Fermi gas are important in studies of macroscopic quantum physics. Both refer to special states of matter in which conspicuous quantum effects appear on a macroscopic scale. Both are quantum degenerate matter. Quantum degeneracy refers to a physical situation in which thermal de-Broglie wavelength of matter wave exceeds inter particle separation. As a result, matter wave properties play a crucial role in determining not only the microscopic nature but also the bulk properties of matter. Particle-particle interaction in degenerate Bose and Fermi gas leads respectively to Bose and Fermi superfluidity.

Let us now discuss some striking similarities as well as differences in BEC and BCS ground states of interacting systems. Let us begin by writing the ground states of uniform interacting systems in momentum space

$$
\begin{array}{ll}
\mathrm{BEC}: & \Psi_{0}^{\mathrm{BEC}}=\prod_{k \neq 0} \phi_{k}=\prod_{k \neq 0} \frac{1}{u_{k}} \sum_{n=0}^{\infty}\left(-\frac{v_{k}}{u_{k}}\right)^{n}\left|n_{k}, n_{-k}\right\rangle \\
\mathrm{BCS}: & \Psi_{0}^{\mathrm{BCS}}=\prod_{k} \psi_{k}=\prod_{k}\left(u_{k}|\mathbf{0}\rangle+v_{k}\left|\mathbf{1}_{k \uparrow}, \mathbf{1}_{-k \downarrow}\right\rangle\right)
\end{array}
$$

where $u_{k}$ and $v_{k}$ are amplitude of corresponding Bose or Fermi quasiparticle associated with celebrated transformation that bears Bogoliubov's name. BEC ground state as expressed in Eq. (II) is a product of all possible nonzero momentum states $\phi_{k}$ which is a coherent superposition of two mutually opposite momentum states $\mathbf{k}$ and $-\mathbf{k}$ occupied by equal number of particles $n$ ranging from zero to infinity. In other words, $\phi_{k}$ is a superposition of all possible pair states $\left|n_{k}, n_{-k}\right\rangle$. All the nonzero momentum states compose the non-condensate part of BEC, while zero-momentum state is the condensate part. Clearly, nonzero momentum states form the structure in the ground state. At zero temperature, non-condensate part consisting of nonzero momentum states arises because of particle-particle interaction. Therefore, we can infer that interaction leads to nontrivial pairing correlation which may be used as a resource for generation of continuous variable entanglement. How to extract this correlation by light scattering and thereby to entangle two spatially separated BECs in number and phase variables by a pair of common laser beams passing through both the condensates has been discussed elsewhere 23. Similar experimental configuration has been recently used to produce and subsequently measure phase difference between two spatially separated BECs [24].

Now let us turn our attention to Eq. (2) which expresses the ground state of an attractively interacting spin-half Fermi system. Figure 1 shows pictorially and very naively what happens to the ground state of noninteracting Fermi system when attractive interaction is switched on. Like BEC ground state, it has a structure that is based on particle-particle pairing (Cooper-pairing) in mutually opposite momentum, 


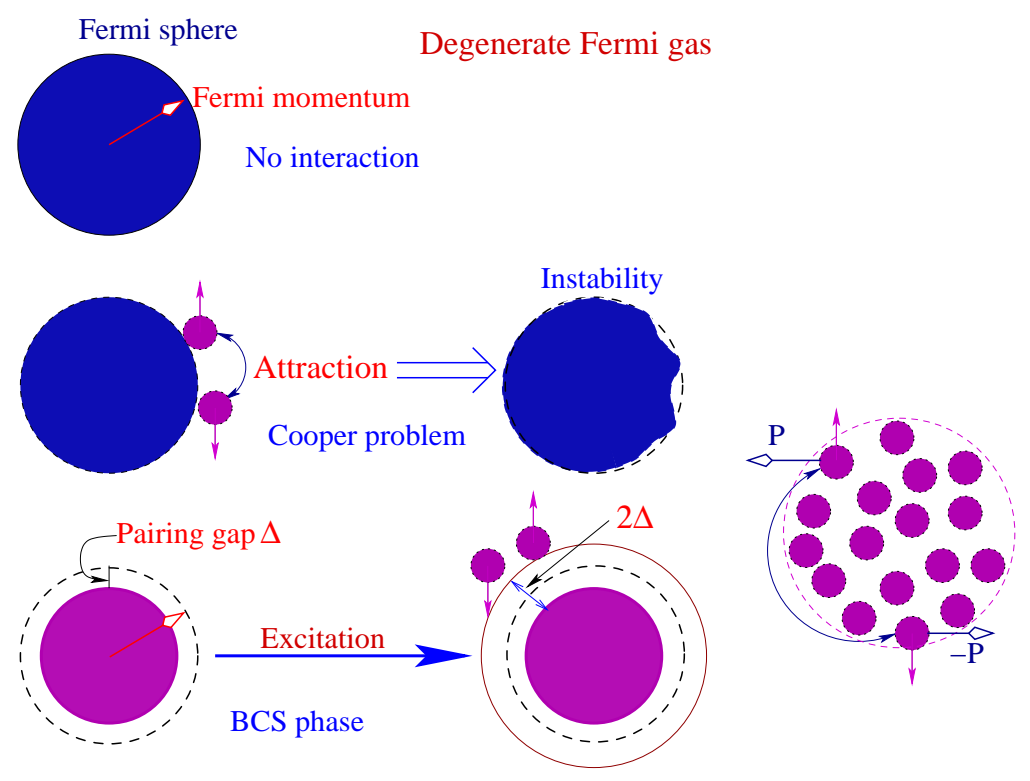

Figure 1. A naive pictorial illustration of Cooper-pair formation and its consequence. For a noninteracting (ideal) Fermi gas, the ground state is simply the Fermi sphere which is completely filled up to Fermi surface and completely empty above the surface. As shown first by Cooper, an attractive inter-fermion interaction, even if it is very weak, leads to formation of an exotic pair-bound state (Cooper-pair) which in turn leads to instability in the Fermi surface. Note that this pairing is basically a manybody effect, since for this effect to occur, quantum degeneracy or near degeneracy is essential. Bardeen, Cooper and Shrieffer then demonstrated that the ground state of a Fermi system with an attractive inter-particle interaction has a gap $\Delta$ which is known as pairing gap. Naively speaking, this ground state forms a sphere in momentum space with a radius which is less than Fermi energy by an amount equal to $\Delta$. To break Coopr-pairs and thereby to excite single-particle excitations, a minimum of $2 \Delta$ energy is required to be imparted on the system. However, various collective modes among which Bogoliubov-Anderson mode is most significant one can be excited below the gap energy. The sphere at the extreme right is to be considered in real space and drawn to illustrate the fact that the two particles whose distance may exceed enormously the average inter-particle separation can form the pairing state.

albeit in opposite spin up $(\uparrow)$ and down $(\downarrow)$ states. The structure of BCS ground state differs from that of BEC because of Pauli's exclusion principle which forbids more than one fermion to occupy a single quantum state. Hence in a uniform Fermi system, there is only one particle having momentum $\mathbf{k}$ and spin up, if it has to form pairing with another particle with opposite momentum and down spin, it will find only one such partner particle. Since pairing occurs in opposite momentum states, the center-of-mass (COM) momentum of a Cooper-pair is zero. Furthermore, the pairing state is in spinsinglet and hence antisymmetric with respect to spin degrees of freedom. Therefore its spatial part must be symmetric. This means pairing must occur in even number of relative angular momentum $l$. In low temperature weak-coupling superconductor, Cooper-pairing occurs in s-wave $(l=0)$ state. Although, a Cooper-pair is a kind of two-particle bound state, it is fundamentally different from familiar bound states like 
diatomic molecule. Cooper-pairing is basically a many-body phenomenon. It occurs only when fermions attract one another under quantum degenerate condition. In contrast a diatomic molecule can be formed by three body interaction. A single molecule can exist in isolation. In contrast, any attempt to isolate a single Cooper-pair from many-body degenerate environment will result in its breaking up into individual fermions. When molecule formation takes place, only nearest neighbor particles form molecular bonding. Cooper-paring can occur between two fermions lying far apart, their distance can greatly exceed average inter-fermion separation. Cooper-pairs can condense into zero (COM) momentum. In fact, a crossover from BCS state of atoms to BEC state of molecules formed from atoms due to a magnetic field Feshbach resonance is an important object of current research interest.

\section{BCS state of trapped Fermi gas of atoms}

To illustrate the main idea, we specifically consider trapped ${ }^{6} \mathrm{Li}$ Fermi atoms in their two lowest hyperfine spin states $\left|g_{1}\right\rangle=\left|2 \mathrm{~S}_{1 / 2}, F=1 / 2, m_{F}=1 / 2\right\rangle$ and $\left|g_{2}\right\rangle=\mid 2 \mathrm{~S}_{1 / 2}, F=$ $\left.1 / 2, m_{F}=-1 / 2\right\rangle$. For s-wave pairing to occur, the atom number difference $\delta N$ of the two components should be restricted by $\frac{\delta N}{N} \leq T_{c} / \epsilon_{F}$ where $T_{c}$ is the critical temperature for superfluid transition and $\epsilon_{F}$ is the Fermi energy at the trap center. Unequal densities of the two components result in interior gap (IG) superfluidity [25, 26]. We have suggested in Ref. [26] that it is possible to experimentally realize IG state in two-component Fermi gas of ${ }^{6} \mathrm{Li}$ atoms by making density mistmatch between the two spin-components. In two remarkable recent experiments [27, 28] using two-component ${ }^{6} \mathrm{Li}$ gas, some results which indicate the occurrence of IG state have been obtained. We here consider only the case $N_{1 / 2}=N_{-1 / 2}$ which is the optimum condition for s-wave Cooper pairing.

Let us consider a cylindrical harmonic trap characterized by the radial (axial) length scale $a_{\perp(z)}=\sqrt{\hbar /\left(m \omega_{\perp(z)}\right.}$. One can define a geometric mean frequency $\omega_{h o}=\left(\omega_{\perp}^{2} \omega_{z}\right)^{1 / 3}$ and a mean length scale by $a_{h o}=\sqrt{\hbar /\left(m \omega_{h o}\right)}$. In Thomas-Fermi local density approximation (LDA) 29, the state of the system is governed by $\epsilon_{F}(\mathbf{r})+V_{h o}(\mathbf{r})+U(\mathbf{r})=$ $\mu$, where $\epsilon_{F}(\mathbf{r})=\hbar^{2} k_{F}(\mathbf{r})^{2} /(2 m)$ is the local Fermi energy, $k_{F}(\mathbf{r})$ denotes the local Fermi momentum which is related to the local number density by $n(\mathbf{r})=k_{F}(\mathbf{r})^{3} /\left(6 \pi^{2}\right)$. Here $U$ represents the mean-field interaction energy and $\mu$ is the chemical potential. At low energy, the mean-field interaction energy depends on the two-body s-wave scattering amplitude $f_{0}(k)=-a_{s} /\left(1+i a_{s} k\right)$, where $a_{s}$ represents s-wave scattering length and $k$ denotes the relative wave number of two colliding particles. In the dilute gas limit $\left(\left|a_{s}\right| k<<1\right), U$ becomes proportional to $a_{s}$ in the form $U(\mathbf{r})=\frac{4 \pi \hbar^{2} a_{s}}{2 m} n(\mathbf{r})$. In the unitarity limit $\left|a_{s}\right| k \rightarrow \infty$, the scattering amplitude $f_{0} \sim i / k$ and hence $U$ becomes independent of $a_{s}$. It then follows from a simple dimensional analysis that in this limit, $U$ should be proportional to the Fermi energy: $U(\mathbf{r})=\beta \epsilon_{F}(\mathbf{r})$ where $\beta$ is the constant. In this limit, the pairing gap also becomes proportional to the Fermi energy.

Under LDA, the density profile of a trapped Fermi gas is given by

$$
n(\mathbf{r})=n(\mathbf{0})\left(1-r_{\perp}^{2} / R_{\perp}^{2}-r_{z}^{2} / R_{z}^{2}\right)^{3 / 2},
$$


where $n(\mathbf{0})=1 /\left(6 \pi^{2} \hbar^{3}\right)[2 m \mu /(1+\beta)]^{3 / 2}$ is the density of the atoms at the trap center. Here $R_{\perp(z)}^{2}=2 \mu /\left(m \omega_{\perp(z)}^{2}\right)$ is the radial(axial) Thomas-Fermi radius. The normalization condition on eq. (3) gives an expression for $\mu=(1+\beta)^{1 / 2}\left(6 N_{\sigma}\right)^{1 / 3} \hbar \omega_{0}$ where $N_{\sigma}$ is the total number of atoms in the hyperfine spin $\sigma$. The Fermi momentum $k_{F}=\left[3 \pi^{2} n(\mathbf{0})\right]^{1 / 3}=(1+\beta)^{-1 / 4} k_{F}^{0}$ where $k_{F}^{0}=\left(48 N_{\sigma}\right)^{1 / 6} / a_{h o}$ is the Fermi momentum of the noninteracting trapped gas.

\section{Stimulated light scattering in Cooper-paired Fermi atoms}

To unravel the nature of Fermi superfluid of atomic gases, it is important to analyze the possible response of this quantum gas due to an external perturbation. A method has been suggested to use resonant light [30] to excite one of the spin components into an excited electronic state and thereby making an interface between normal and superfluid atoms as in superconductive tunneling. This has a threshold equal to the gap energy $\Delta$. This method has been applied in recent experiments [10, 31] with the use of rf field for estimating gap energy. There have been a number of proposals [30, 32] for probing pairing gap.

We calculate response function of superfluid Fermi gas due to stimulated light scattering that does not cause any electronic excitation in the atoms. We particularly emphasize the role of light polarization in single-particle excitations which have a threshold $2 \Delta$. We present a scheme by which it is possible to have single-particle excitation in only one partner atom (of a particular hyperfine spin state) of a Cooperpair using proper light polarizations in the presence of a magnetic field. This may lead to better precision in spin-selective time-of-flight detection of scattered atoms. Furthermore, spin-selective light scattering allows for unequal energy and momentum transfer into the two partner atoms of a Cooper-pair. This may be useful in exciting Bogoliubov-Anderson (BA) phonon mode of symmetry breaking by making small difference in momentum transfers received by the two partner atoms from the photon fields. Recently, a number of authors [33, 34, 35] have studied Bogoliubov-Anderson (BA) mode [36, 37, 38, in fermionic atoms as a signature of superfluidity. BA mode is associated with long wave Cooper-pair density fluctuations. In electronic superconductor, this mode is suppressed due to long wave Coulomb interaction. In neutral superfluid Fermi system such as trapped atomic Fermi gas, this mode is well defined and should in principle be experimentally observable. However, its experimental detection poses a challenging problem since it is a near zero-energy zero-momentum mode.

Figure 2 shows the schematic level diagram for stimulated light scattering by twocomponent ${ }^{6} \mathrm{Li}$ atoms in the presence of an applied magnetic field which is tuned near the Feshbach resonance ( $\sim 834$ Gauss) results in strong inter-component s-wave interaction. At such high magnetic fields, the splitting between the two ground hyperfine states is $\sim 75 \mathrm{MHz}$ while the corresponding splitting between the excited states $\left|e_{1}\right\rangle=\mid$ $\left.2 \mathrm{P}_{3 / 2}, F=3 / 2, m_{F}=-1 / 2\right\rangle$ and $\left|e_{2}\right\rangle=\left|2 \mathrm{P}_{3 / 2}, F=3 / 2, m_{F}=-3 / 2\right\rangle$ is $\sim 994 \mathrm{MHz}$. 
Two off-resonant laser beams with a small frequency difference are impinged on atoms, the scattering of one laser photon is stimulated by the other photon. In this process, one laser photon is annihilated and reappeared as a scattered photon propagating along the other laser beam. The magnitude of momentum transfer is $q \simeq 2 k_{L} \sin (\theta / 2)$, where $\theta$ is the angle between the two beams and $k_{L}$ is the momentum of a laser photon. Let both the laser beams be $\sigma_{-}$polarized and tuned near the transition $\left|g_{2}\right\rangle \rightarrow\left|e_{2}\right\rangle$. Then the transition between the states $\left|g_{1}\right\rangle$ and $\left|e_{2}\right\rangle$ would be forbidden while the transition $\left|g_{1}\right\rangle \rightarrow\left|e_{1}\right\rangle$ will be suppressed due to the large detuning $\sim 900 \mathrm{MHz}$. This leads to a situation where the Bragg-scattered atoms remain in the same initial internal state $\left|e_{2}\right\rangle$. Similarly, atoms in state $\left|g_{1}\right\rangle$ only would undergo Bragg scattering when two $\sigma_{+}$polarized lasers are tuned near the transition $\left|g_{1}\right\rangle \rightarrow\left|2 \mathrm{P}_{3 / 2}, F=3 / 2, m_{F}=3 / 2\right\rangle$. Thus, we infer that in the presence of a high magnetic field, it is possible to scatter atoms selectively of either spin components only by using circularly polarized Bragg lasers. We assume that both the laser beams are $\sigma_{-}$polarized and tuned near the transition $\left|g_{2}\right\rangle \rightarrow\left|e_{2}\right\rangle$. Under such conditions, considering a uniform gas of atoms, the effective laser-atom interaction Hamiltonian 22] in electric-dipole approximation is $H_{I} \propto \sum_{\mathbf{k}, \sigma=1,2} \gamma_{\sigma \sigma} \hat{c}_{\sigma}^{\dagger}(\mathbf{k}+\mathbf{q}) \hat{c}_{\sigma}(\mathbf{k})$, where $\hat{c}_{\sigma}(k)$ represents annihilation operator of an atom with momentum $\mathbf{k}$ in the internal state $\sigma$. The subscript $\sigma=1(2)$ refers to the state $\left|g_{1}\right\rangle\left(\left|g_{2}\right\rangle\right)$. The bare vertex $\gamma_{\sigma \sigma}$ is given by the Kramers-Heisenberg formula [39]

$$
\gamma_{\sigma \sigma}=\frac{e^{2} \mathcal{E}_{1} \mathcal{E}_{2}}{m_{e} \sqrt{n} \hbar \omega_{1} \omega_{2}} \sum_{i=1,2} \frac{\left(\mathbf{d}_{\sigma \sigma} \cdot \hat{\mathcal{E}}_{2}\right)\left(\mathbf{d}_{\sigma \sigma} \cdot \hat{\mathcal{E}}_{1}\right)}{\hbar^{2}\left(\omega_{\sigma \sigma}-\omega_{i}\right)}
$$

where $d_{i i}$ is the dipole matrix element between $|g\rangle_{i}$ and $|e\rangle_{i}$ and $n$ is the incident photon number which is assumed to be equal for both the laser beams. Here $m_{e}$ and $e$ are the mass and charge, respectively, of the valence electron; $\hat{\mathcal{E}}_{i}$ and $\omega_{i}$ represent the electric field and frequency, respectively, of $i$-th laser beam and $\omega_{\sigma \sigma}$ is the atomic frequency between the states $|g\rangle_{\sigma}$ and $|e\rangle_{\sigma}$. For the particular case of $\sigma_{-}$polarization in the presence of magnetic field as discussed above, one finds $\gamma_{22}>>\gamma_{11}$. On the other hand, in the absence of magnetic field, one has $\gamma_{11} \simeq \gamma_{22}$.

\subsection{The response function}

We assume that, except the center-of-mass momentum, the spin or any other internal degrees of atom does not change due to light scattering.

Now, one can define the density operators by $\rho_{q}^{(0)}=\sum_{\sigma, \mathbf{k}} a_{\sigma, \mathbf{k}+\mathbf{q}}^{\dagger} a_{\sigma, \mathbf{k}}$ and

$$
\rho_{q}^{(\gamma)}=\sum_{k, \sigma} \gamma_{\sigma \sigma} a_{\sigma, \mathbf{k}+\mathbf{q}}^{\dagger} a_{\sigma, \mathbf{k}}
$$

One can identify the operator $\rho_{q}^{(0)}$ as the Fourier transform of the density operator in real space. The scattering probability is related to the susceptibility

$$
\chi\left(\mathbf{q}, \tau-\tau^{\prime}\right)=-\left\langle T_{\tau}\left[\rho_{q}^{(\gamma)}(\tau) \rho_{-q}^{(\gamma)}\left(\tau^{\prime}\right)\right]\right\rangle .
$$

where $T_{\tau}$ is the complex time $\tau$ ordering operator and $\langle\cdots\rangle$ means thermal averaging. 
Pair-correlation in Bose-Einstein Condensate and Fermi Superfluid of Atomic Gases 8

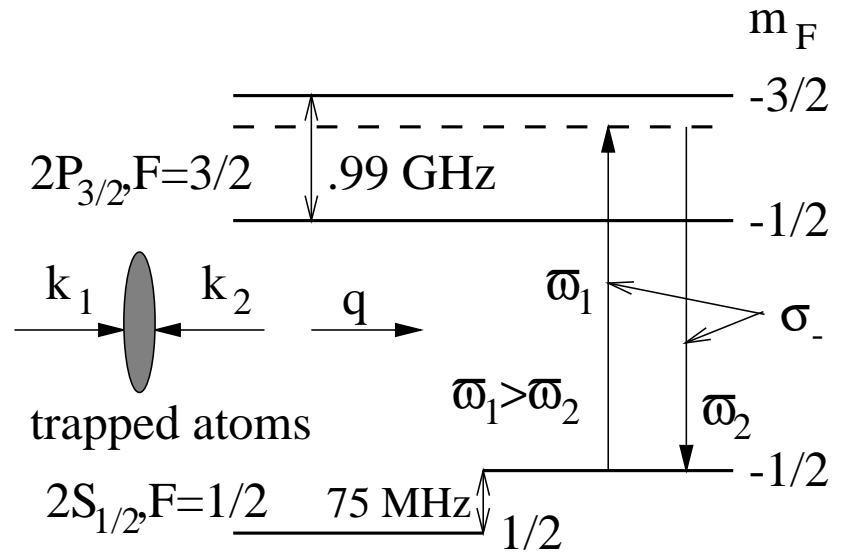

Figure 2. A schematic level diagram for polarization-selective light scattering in two-component Fermi gas of ${ }^{6} \mathrm{Li}$ atoms

The dynamic structure factor is related to $\chi$ by $\chi\left(\mathbf{q}, \omega_{n}\right)$ as

$$
S(\mathbf{q}, \omega)=-\frac{1}{\pi}\left[1+n_{B}(\omega)\right] \operatorname{Im}\left[\chi\left(\mathbf{q}, \mathrm{z}=\omega+\mathrm{i} 0^{+}\right)\right] .
$$

This follows from generalized fluctuation-dissipation theorem. In order to treat collective excitations, it is essential to go beyond Hartree approximation and apply either a kinetic equation or a time-dependent Hartree-Fock equation or a random phase approximation 38. The essential idea is to take into account the residual terms which are neglected in the BCS approximation and thereby treat the off-diagonal matrix elements (vertex functions) of single-particle operators in a more accurate way [40, 38].

The detailed method of calculation of response function of superfluid Fermi atoms due to stimulated light scattering is given elsewhere [22].

We here present the final result

$$
\chi(\mathbf{q}, \omega)=2 N(0) \gamma_{0}^{2}\langle B\rangle+2 N(0)\left[\langle A\rangle+\frac{\omega^{2}\langle f\rangle^{2}}{4 \Delta^{2}\left\langle\beta^{2} f\right\rangle}\right] \gamma_{3}^{2}
$$

where $N(0)$ is the density of states at the Fermi surface and

$$
A=\frac{\left(\mathbf{v}_{k} \cdot \mathbf{p}_{q}\right)^{2}-\omega^{2} f}{\omega^{2}-\left(\mathbf{v}_{k} \cdot \mathbf{p}_{q}\right)^{2}}, \quad B=\frac{\left(\mathbf{v}_{k} \cdot \mathbf{p}_{q}\right)^{2}(1-f)}{\omega^{2}-\left(\mathbf{v}_{k} \cdot \mathbf{p}_{q}\right)^{2}} .
$$

Here $\mathbf{p}_{q}=\hbar \mathbf{q}$ and $\mathbf{v}_{k}$ is the velocity of the atoms with momentum $\mathbf{k}, f(q)=$ $\sin ^{-1}(\beta) /\left[\beta\left(1-\beta^{2}\right)^{1 / 2}\right]$ and $\beta^{2}=\left[\omega^{2}-\left(\mathbf{v}_{k} \cdot \mathbf{p}_{q}\right)^{2}\right] /\left(4 \Delta^{2}\right)$. The symbol $\langle X\rangle$ implies averaging of a function $X$ over the chemical potential surface: $\langle X\rangle=$ $[N(0)]^{-1} \int d^{3} \mathbf{k} \delta\left(\epsilon_{k}\right) X$. Note that the Eq. (8) applies to the single-particle excitation regime $\left(\beta^{2}>1\right)$ only.

\section{Results and discussions}

Figure 3 shows $S(\omega, \mathbf{q})$ as a function of $\omega$ for a uniform and trapped gas for different values of $a_{s}$. In the case of trapped gas, we use LDA with local chemical potential $\mu(\mathbf{r})$ determined from equation of state of interacting Fermi atoms in a harmonic trap. 

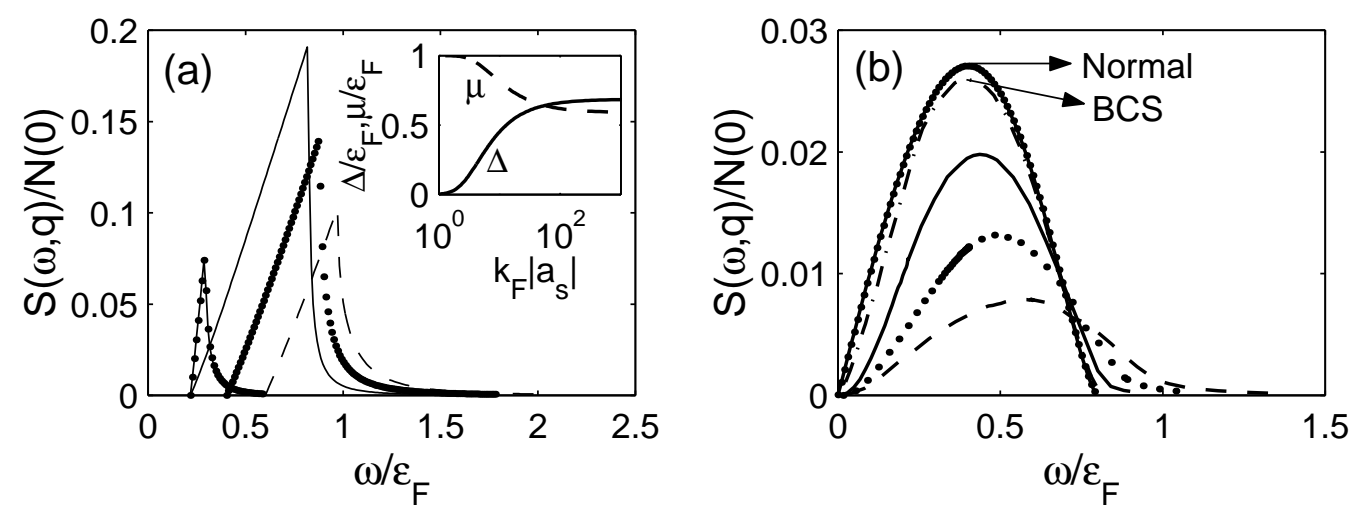

Figure 3. (a) Dimensionless DSF $S(\omega, \mathbf{q}) / N(0)$ for single-particle excitations of a uniform superfluid Fermi gas is plotted as a function of dimensionless energy transfer $\omega / \epsilon_{F}$ for different values of the scattering length $\left|a_{s}\right|=2.76 k_{F}^{-1}$ (solid), $\left|a_{s}\right|=3.89 k_{F}^{-1}$ (dotted), $\left|a_{s}\right|=5.47 k_{F}^{-1}$ (dashed) for a fixed momentum transfer $q=0.8 k_{F}$. The dash-dotted curve is plotted for $\left|a_{s}\right|=2.76 k_{F}^{-1}$ and $q=0.4 k_{F}$. The inset to Fig. (a) shows the variation of the gap $\Delta$ and the chemical potential $\mu$ as a function of $\left|a_{s}\right|$. For large $a_{s}, \mu$ and $\Delta$ saturate at $0.59 \epsilon_{F}$ and $0.68 \epsilon_{F}$, respectively. (b) Same as in Fig. (a) but for a trapped superfluid Fermi gas for a fixed momentum transfer $q=0.8 k_{F}$ $\left(k_{F}\right.$ refers to the Fermi momentum at the trap center). Also shown are the DSF for small $\Delta=0.05$ (BCS) and $\Delta=0$ (normal).

When $a_{s}$ is large, the behavior of $S(\delta, \mathbf{q})$ is quite different from that of normal as well as weak-coupling BCS superfluid. This can be attributed to the occurrence of large gap for large $a_{s}$. In contrast to the case of a uniform superfluid, $S(\delta, \mathbf{q})$ for a superfluid trapped Fermi gas has a structure below $2 \Delta(0)$, where $\Delta(0)$ is the gap at the trap center. As the energy transfer decreases below $2 \Delta(0)$, the slope of $S(\delta, \mathbf{q})$ gradually reduces. Particularly distinguishing feature of $S(\delta, \mathbf{q})$ of a superfluid compared to normal fluid is gradual shift of the peak as $a_{s}$ or $\Delta$ increases. The quasiparticle excitations occur only when $2 \Delta(\mathbf{x})<\omega$. This implies that, when $\omega$ is less than $2 \Delta(0)$, the atoms at the central region of the trap can not contribute to quasiparticle response.

\section{Conclusion}

In conclusion, we have studied long-ranged pair-correlation inherent in both the BEC and BCS states. We have also investigated polarization-selective light scattering in Cooper-paired Fermi atoms as a means of estimating the gap energy. Our results suggest that it is possible to detect the pairing gap by large-angle (i.e., large $q$ ) Bragg scattering. Small angle polarization-selective stimulated light scattering may be useful in exciting BA mode. The pair-correlation which may be a generic feature of all macroscopic quantum systems with long-range order may serve as a potential resource for mayparticle robust entanglement that is central to quantum information science. 
Pair-correlation in Bose-Einstein Condensate and Fermi Superfluid of Atomic Gases10

\section{References}

[1] Anderson M., Ensher J. R., Matthews M. R., Wieman C. E., and Cornell E. A. 1995 Science 269 198; Bradley C. C., Sackett C. A., Tollett J. J. , and Hulet R. G. 1995 Phys. Rev. Lett. 751687 ; Davis K. B. , Mewes M. O., Andrews M. R., van Druten N. J., Durfee D. S., Kurn D. M. , and Ketterle W. 1995 Phys. Rev. Lett. 753969

[2] B. DeMacro and D. S. Jin 1999 Science 2851703

[3] A. G. Truscott, K. E. Strecker, W. I. McAlexander, G.B. Patridge, and R. G. Hulet 2001 Science 2912570

[4] S. R. Granade, M. E. Gehm, K. M. O'Hara, and J. E. Thomas 2002 Phys.Rev.Lett. 88, 120405; O'Hara et al. 2002 Science 2982179

[5] Zwierlein M. W., Abo-Shaeer J. R., Schirotzek A., Schunck C. H., Ketterle W. 2005 Nature 435 1047

[6] F. Schreck et al. 2001 Phys. Rev. Lett. 87 080403;T. Bourdel et al. 2003 ibid. 91020402

[7] Z. Hadzibabic et al., Phys. Rev. Lett. 88, 160401 (2002).

[8] G. Roati, F. Riboli, G. Modungo, and M. Inguscio, Phys. Rev. Lett. 89, 150403 (2002).

[9] Jochim S. et al. 2003 Science 3022101

[10] Cin C. et al. 2004 Science 3051128

[11] Greiner M., Regal C. A., and Jin D. S. 2005 Phys. Rev. Lett. 94070403

[12] Kinast, J. et al. 2004 Phys. Rev. Lett. 92150402

[13] Bartenstein M. et al. 2004 Phys. Rev. Lett. 92203201

[14] Stringari S. 2004 Europhys. Lett. 65749

[15] Noziéres P. and Schmitt-Rink S. 1985 J. Low. Temp. Phys. 59195

[16] Sa de Melo C.A.R., Randeria M. and Engelbrecht J.R. 1993 Phys. Rev. Lett. 71 3202; Engelbrecht J.R., Randeria M. and Sa de Melo C.A.R. 1997 Phys. Rev. B 5515153

[17] Holland M., Kokkelmans S. J. J. M. F., Chiofalo M. L. and Wasler R. 2001 Phys. Rev. Lett. 87 120406; Timmermans E. et al. 2001 Phys. Lett A 285 228; Ohashi Y. and Griffin A. 2002 Phys. Rev. Lett. 89 130402; Hofstetter W. et al. 2002 Phys. Rev. Lett. 89220407

[18] Greiner M., Regal C. A. and Jin D. S. 2003 Nature 426 537; Jochim S. et al. 2003 Science 302 2101; Zwierlein M. W. et al. 2003 Phys. Rev. Lett. 91250401

[19] Modugno G. et al. 2002 Science 297 2240; Strecker K. E. et al. 2003 Phys. Rev. Lett. 91080406 ; Cubizolles J. et al. 2003 Phys. Rev. Lett. 91240401

[20] Falco G. M. and Stoof H. T. C. 2004 Phys. Rev. Lett. 92 130401; Carr L. D., Shlyapnikov G. V. and Castin Y. 2004 Phys. Rev. Lett. 92 150404; Heiselberg H. 2003 Phys. Rev. A 68 053616, Perali A., Pieri P. and Strinati G. C. 2003 Phys. Rev. A 68 031601; Perali A., Pieri P., Pisani L. and Strinati G. C. lanl e-print cond-mat/0311309

[21] Deb B. and Agarwal G. S., 2002 Phys.Rev.A 65063618.

[22] Deb B., 2006 J. Phys. B: At. Mol. \& Opt. Phys. 39, 529.

[23] Deb B. and Agarwal G. S., 2003 Phys.Rev.A 67023603.

[24] Saba M., Pasquini T. A., Sanner C., Shin Y., Ketterle W., Pritchard D. E., 2005 science 3071945.

[25] W. V. Liu and F. Wilczek 2003 Phys.Rev.Lett. 90047002

[26] Deb B., Mishra A., Mishra H. and Panigrahi P. K. 2004 Phys. Rev. A 70011604

[27] Zwierlein M. W., Schirotzek A., Schunck C. H., and Ketterle W, 2006 Science 311492

[28] Partridge G. B., Lui W., Kamar R. I., Liao Y., and Hulet R. G., 2006 Science 311503

[29] Houbiers M. et al. 1997 Phys. Rev. A 56 4864; Vichi L. and Stringari S. 1999 Phys. Rev. A 60 4734

[30] Törmä P. and Zoller P. 2000 Phys. Rev. Lett. 85 487; Bruun G. M. et al. 2001 Phys. Rev. A 64 033609; Kinnunen J., Rodriguez M., and Törmä P 2004 Phys. Rev. Lett. 92 230403; Bruun G. M. and Baym G. 2004 Phys. Rev. Lett. 93 150403; Büchler H. P., Zoller P., Zwerger W. 2004 Phys. Rev. Lett., 93080401

[31] Kinnunen J., Rodriguez M., and Törmä P 2004 Science 3051131 
[32] Zhang W., Sackett C. A. and Hulet R. G. 1999 Phys. Rev. A 60 504; Ruostekoski J. 1999 Phys. Rev. A 60 1775; Rodriguez M. and Törmä P. 2002 Phys. Rev. A 66033601.

[33] Bruun G. M. and Mottelson B. R. 2001 Phys. Rev. Lett. 87270403

[34] Ohashi Y. and Griffin A. 2003 Phys. Rev. A 67 063612; Ohashi Y. and Griffin A. lanl archive cond-mat/0503641

[35] Minguzzi A., Ferrari G. and Castin Y. 2001 Eur. Phys. J. D. 1749

[36] Bogoliubov N. N. 1958 Nuovo Cimento 7 6; Bogoliubov N. N., Tolmachev V. V., and Shirkov D. V. 1959 A New Method in the Theory of Superconductivity (Consultants Bureau, NY).

[37] Anderson P. W. 1958 Phys. Rev. 1121900

[38] Martin P. C., in 1969 Superconductivity, Vol.1, edited by Parks R. D. (Dekker, NY).

[39] Sakurai J. J. 1967 Advanced Quantum Mechanics (Pearson Education, Inc.)

[40] Schrieffer J. R. 1964 Theory of Superconductivity ( W. A. Benjamin) 